\title{
Deblurring Method for Motion Blurred Images based on GAN
}

\author{
Ning Li, Songnan Chen, Mengxia Tang, Jiangming Kan* \\ School of technology, Beijing Forestry University \\ No.35 Tsinghua East Road, Haidian District, Beijing, 10086. \\ China
}

Received: July 10, 2020. Revised: January 9, 2021. Accepted: February 8, 2021. Published: February 12, 2021.

\begin{abstract}
The purpose of image motion deblur is to recover the underlying clear image from the corresponding blur image. In most traditional methods, the image recovery task is formulated as a problem of blur core estimation and use a priori to calculate. In this paper we proposes a generative adversarial network(GAN) model based on the mobilenet-V3 network structure to meet the needs of motion blurred image recovery on mobile devices. Based on traditional evaluation indicators, we propose a new evaluation metric on mobile device. Extensive experiments show that our method is superior to the competing methods.
\end{abstract}

Keywords-Motion deblur, generative adversarial network, mobilenet-V3 networks, image recovery.

\section{INTRODUCTION}

$\mathrm{T}$ hen application of motion blurred image restoration has been extended many scientific and technical areas, such as astronomical observation, remote sensing, military, medical imaging, biological research, transportation, history, humanities, industrial vision, image video, and other fields, thus it is important to research on the motion blurred image restoration methods.

Image motion blur occurs in the process of camera exposure, as a result of relative movement between camera and objects. Motion blur can be divided into non-uniform blur and uniform blur. Non-uniform blur is local blur and caused by the movement of object at exposure time. Uniform blur is global blur and caused by camera movements[1]. Motion blur is also called dynamic blur which has a motion trajectory. Theoretically, motion blurred image can be treated as the synthesis of a clear image and motion trajectory. The formula is expressed as shown in Eq.1.

$$
I_{B}=k(M) * I_{s}+N
$$

where $I_{B}$ is the blurred image, $K(M)$ is the blur kernel determined by motion trajectory $M . I_{S}$ is the latent clear image, $*$ is the convolutional operator, $N$ is the additive noise. For non-uniform blur, different pixels has different blur kernels, while for uniform blur, kernels of each pixel are similar. The task of image restoration is to estimate the latent clear image $I_{S}$ and the blur kernel $K(M)[1]$. For most of the cases, clear image $I_{S}$ and blur kernel $K(\mathrm{M})$ are unknown, so image deblurring is an ill-posed problem and the clear image and the blur kernel cannot directly obtain. Most of the classic methods are utilizing the distribution probability to construct image priors and thus estimate posterior probability of the clear image and the blur kernel. In addition, the basic principle of deep learning network is to study and predict probability distribution of the training objects, which makes it suitable for image deblurring task.

In the past decades, various natural images priors and kernel priors have been used to approximate the solution space of the latent sharp image, including heavy-tailed gradient prior, sparse kernel prior, gradient prior, normalized sparsity and dark channel prior[39,40]. But these image priors cannot adapt to complex environments, thus resulting in under-deblurring (deblurred image are still blurred) and over-deblurring (deblurred image has artifacts) [2-6]. With the rapid development of deep learning techniques in recent years, convolution neural network (CNN) has been used in many areas. A main way of the application of $\mathrm{CNN}$ in image deblurring is to utilize CNN to fit the unknown $K(M)$ and thus obtain clear image $I_{S}$ [1]. Meanwhile, with the wide use of mobile devices such as mobile phone, how to utilize deep network to obtain sharp image for devices with limited computing power and low energy consumption requirements should be considered. In this paper, we aim at proposing image deblurring methods for mobile device.

Classic methods usually construct sharp image prior to estimate motion blur kernel for solving the deblurring problem. Typical image priors including sparse gradient [6-9], normalized sparsity [10], color line model [27], L 0 gradient [11], image patch prior[15] and self-similarity[16]. Although these image priors are effective for natural image deblurring, but may fail on some specific cases such as text, face and low illumination image, as these images have different statistical characteristics with natural images. To deal with these cases, Pan et al.[12] proposed to utilize image intensity and L0 
gradient [13]prior to deblur text image. Hu et al.[11] estimated the blurring kernel by detecting light stripes in low light image. Pan et al. [17] proposed a dark channel prior for image deblurring and showed effective for face, text and low light image, but may fail when there are no dark channel pixels. Yan et al.[18] proposed to utilize extreme channel for image deblurring. But for most of the artificially selected priors, they cannot capture the complex blur changes in real images, the emerging deep learning technique promotes the breakthrough of image restoration task. With deep learning networks, there appeared many deblurring approaches. Schuler et al. [28] proposed a non-blind deblurring method by estimating the blur kernel with a CNN network, and recover the latent clear image with traditional method. Sun et al. [20] proposed a sequential blur restoration method, they utilized 73 potential blur kernels to produce blurred and sharp image pairs, then they trained a classification $\mathrm{CNN}$ to estimate the probability of the blur kernel in each local patches. The network is trained by the optimization of an energy function consists of a CNN likelihood function and a smooth prior function, then recover the latent clear image by a traditional method [26]. Li et al. [25] proposed a data-driven discriminant priori based deblurring method, they described image prior as a binary classifier implemented by $\mathrm{CNN}$, which can identify whether the input image is clear or not, and then embedded it in the MAP framework, which facilitates the blind deblurring in various scenes. Gong et al. [24] utilized fully convolutional network to estimate movement flow. Chakrabarti [22] trained a deep network to predict the Fourier parameters of the deconvolution filter. All the above methods have not deviate from traditional deblurring methods and require a precise kernel estimation step to restore the potential clear image. With the success of deep learning, the blur restoration network has developed from blur kernel estimation based method to the end-to-end way which can directly recover the latent clear image through the deep network. Nah et al. [19] proposed a multiscale CNN which can deblur images obtained by different sources in an end-to-end manner. Tao et al. [32] expanded multiscale CNN from [14,19] to scale recursive $\mathrm{CNN}$ and thus obtain a better result. The success of Generative Adversarial Networks (GAN), it has been wildly used in image enhancement, semantic segmentation, style transfer and other fields.

Inspired by the utilization of GAN for image translation, many authors regard image restoration as a special case of image translation and proposed many deblurring methods based on GAN. Both supervised and unsupervised methods have developed significantly in recent years. Ramakrishnan et al. [29] inspired by the utilization of pix2pix framework in image translation [34], they used the combination of a pix2pix framework and a densely connected convolutional network [15] to perform blind image deblurring. Kupyn et al. treated deblurring task as a special case of image translation [30] problem and proposed a deblurGAN model [23] by improving cGAN to Wasserstein GAN. With the success of deblurGAN, a DeblurGAN-v2 [31] is proposed by changing the backbone network to feature pyramid network (FPN), thus the memory and the computation are reduced, while the deblurring performance and efficiency are improved. It mainly utilized two backbone networks for feature extraction. One is
Inception-ResNet-v2 network [35] to obtain better restoration results; another is a lightweight network [21,37] to obtain better efficiency.

In the unsupervised field, Nimisha et al.[36] proposed an unsupervised image recovery method based on GAN model and added the blur loss and multi-scale gradient loss for training. $\mathrm{Lu}$ et al.[33] utilized separate representation[34] based on the realization of different image translation tasks, an unsupervised domain specific image deblurring method based on unentangled representation was proposed, better results are obtained both on real-world dataset and synthetic dataset.

In this paper, we modified the newest DeblurGAN-v2 model, and used the newest lightweight network Mobilenet-V3 as features extraction backbone network, which provides better results both for running time and computing costs. And the compromise rate as a new evaluation index was proposed to evaluate the accuracy and the calculation cost for the performance of the proposed model.

\section{DEBLURRING METHODS}

In this section, the basic principles, network structure, loss function, training details and data set pre-processing will be discussed in details. We improved the DeblurGAN-v2 network by modifying the backbone network of feature extraction. The overall network is an end-to-end generative adversarial network based on CGAN which consists of a generator and two discriminators, the two discriminators perform global and local discriminations on two scales respectively. The training process was conducted on paired data sets in a supervised manner, finally the generator can directly output the restored clear image when a blurred image is input.

\section{A. Network structure}

As shown in Fig.1, the network is a two stream framework which consists of a generator and two discriminators. Feature pyramid structure (FPN) is used in the generator part; the backbone network of this structure can be altered. Relative discriminator is adopted and both the global and the local minimum mean square error loss are used in training.

The generator network. In 2017, Lin et al. [38] proposed the FPN to deal with the feature extraction of target detection problems. Analyzing the process of feature extraction, the lower layer contains less feature semantic information, but the target location is accurate, and the higher layer contains rich feature semantic information, but the target location is not accurate enough. If there is only a single high-level feature, the target location information will be lost. If the classic pyramid image method is used for multi-scale change enhancement, this will bring a huge amount of calculation. The goal of FPN is to construct a feature pyramid network structure with a very small amount of calculation. The feature pyramid structure used for fuzzy restoration is composed of an FPN backbone network, as shown in Fig. 2. In the left part, a bottom-up process is conducted through convolutional layers and pooling layers, features are obtained as 
enc0-enc4. In the right part, an top-down up-sampling is conducted, since the location information is not accurate after up-sampling and down-sampling, it is necessary to construct a horizontal connection between the reconstructed layer and the corresponding feature map, thus can output accurate location. Five final feature maps with different scales, MAP0-MAP4, are obtained as outputs. The outputs are up-sampled to a quarter of the input size and connected into a tensor which contains different levels of semantic information. At the end of the network, an up-sampling layer and a convolutional layer are added to recover the clear image and remove artifacts.

The backbone network of the generator which extracts features adopts the model based on ImageNet pre-training model (including more semantically related features).
Compared to Mobilenet-v2 network which used in DeblurGAN-v2, in our proposed method, the newest Mobilenet-V3 network is used. We added the SE structure to the model and placed it after deep convolution. Because the SE structure consumes a certain amount of time, we change the number of channels of the expansion layer to $1 / 4$ of the original in the structure containing SE. This not only improves accuracy, but also does not increase time consumption. The comparison of the mobilenet-v3 and Mobilenet-v2 network tails is shown in Fig.3. Two structures of Mobilenet-v3 are proposed which are Mobilenet-v3 large and Mobilenet-v3 small which are suitable for situations with different requirements on resources respectively.

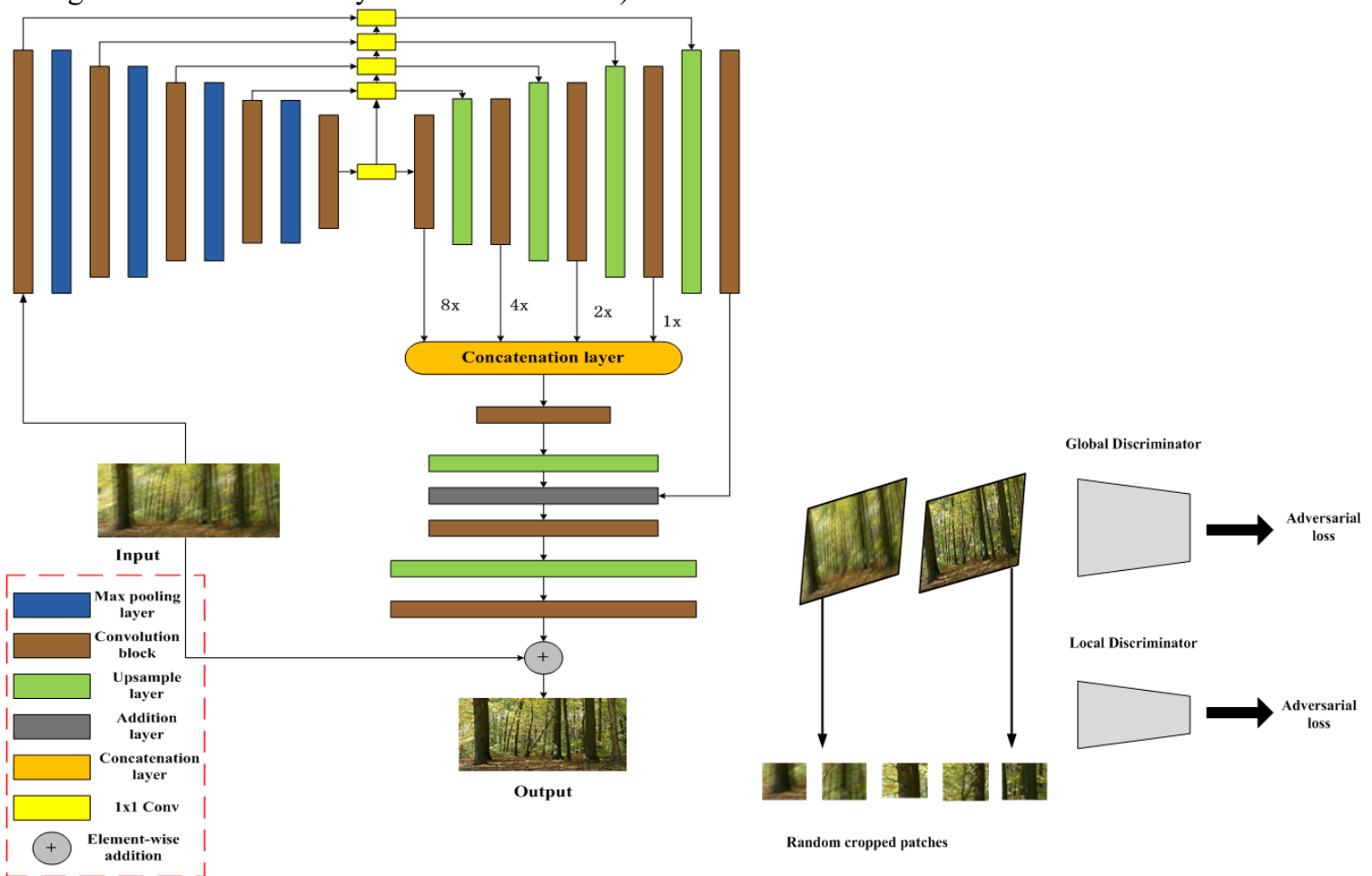

Fig.1. The overall network structure

Mobilenet-v3 small improved the accuracy of ImageNet classification tasks by about 3.2 percent over Mobilenet-v2, and improved the reasoning efficiency by 15 percent. Mobilenet-v3 large improved the accuracy of ImageNet classification tasks by about 4.6 percent over V2, and reasoning efficiency by 5 percent. Mobilenet-v3 Large achieved the same accuracy on COCO as V2, with a $25 \%$ increase in reasoning efficiency. Besides, in this paper, a mobilenet-V3-DSC network is proposed by modifying mobilenet-V3 network to further reduce the network size and computing power. 


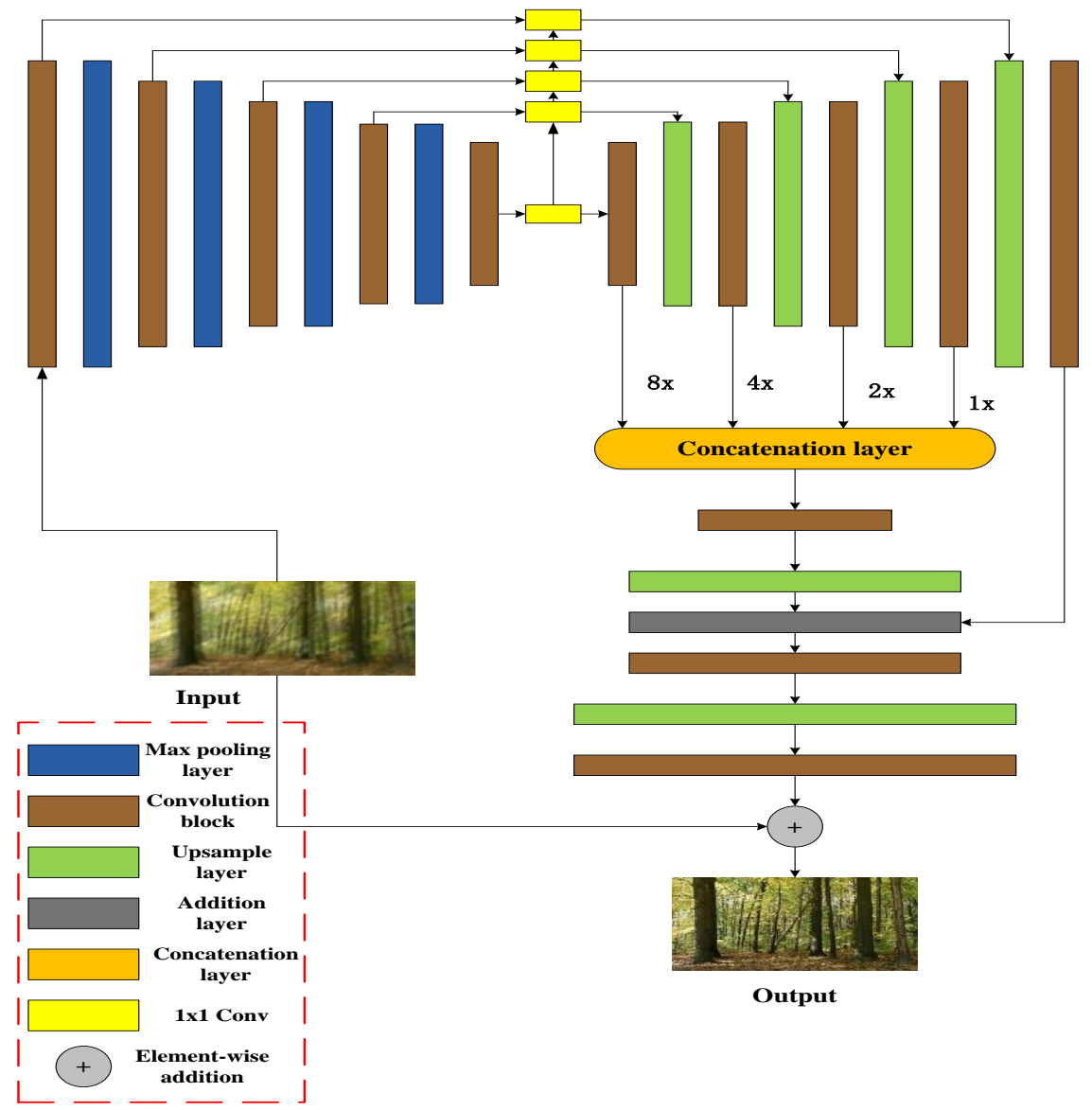

Fig.2. Feature pyramid structure of backbone network.
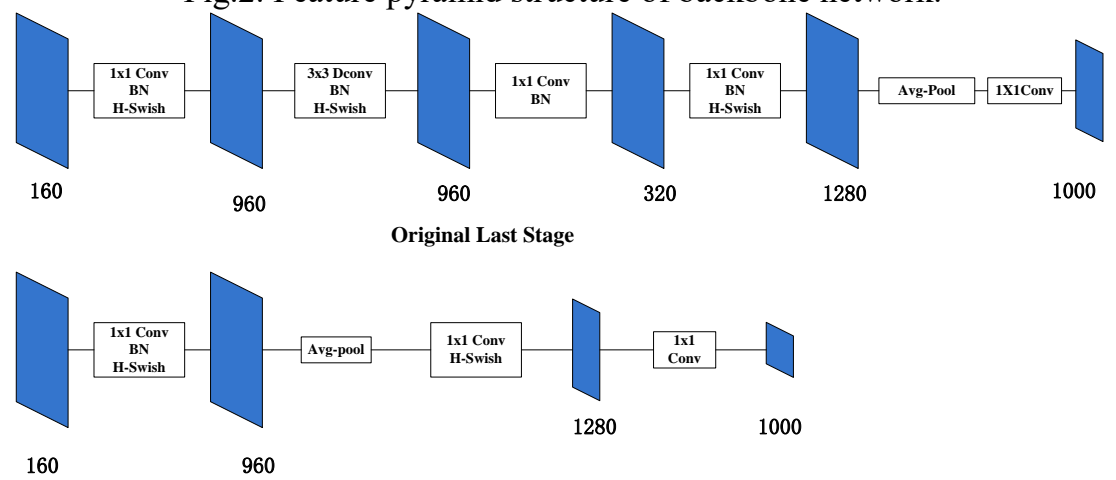

Efficient Last Stage

Fig.3 The difference between Mobilenet-v3 and Mobilenet-v2

The discriminator. We uses a double-scale discriminator structure, as shown in Fig. 4. PatchGAN discriminator is used to distinguish image patches. Besides, a global discriminator is introduced as well. 


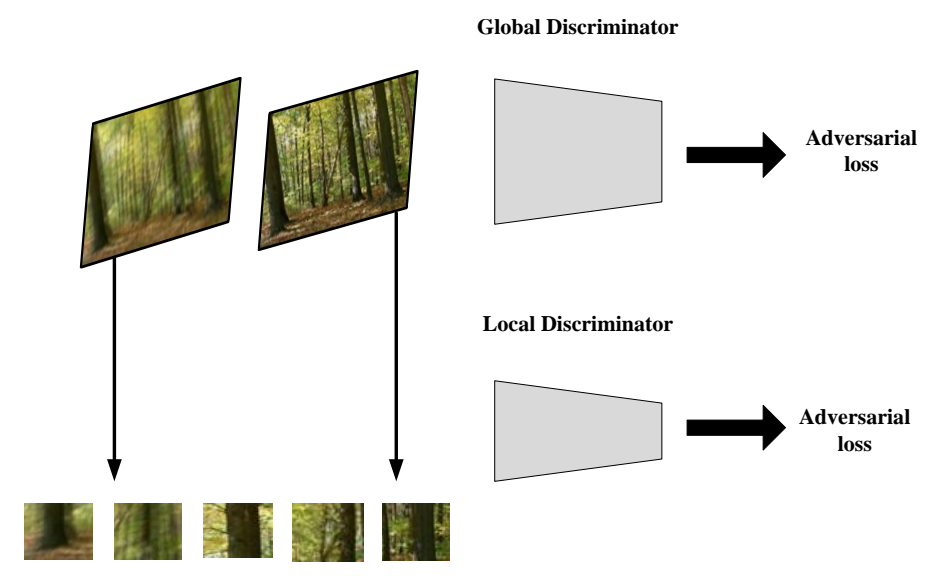

Random cropped patches

Fig. 4. Discriminator structure

\section{B. Data processing and experimental details}

Data processing.In this paper, GoPro data set [18] was used to train the network, which acquired the 240FPS video with GOPRO4 HERO Black camera, and then averaged the images with consecutive 7 to 13 frames to obtain different degrees of blur. The shutter speed of each clear image is $1 / 240$ s. Averaging 15 frames is equivalent to obtaining a blurry image with a shutter speed of $1 / 16 \mathrm{~s}$. The frame in the middle is taken as the clear image corresponding to the blurred image. Finally, 3214 pairs of blurred and clear image with resolution of $1280 \times 720$ are generated. The blurred images in the GoPro data set were averaged out from the continuous clean images, which resulted in distortion of the blurred images. Therefore, the video frame interpolation model [19] was used to increase the original 240FPS video to 3840FPS, and then average was performed within the same time window to obtain a smoother and continuous blurred image.

Experimental details. Similar to previous related work $[1,11]$, the whole network is trained by two steps, first, the backbone network is pre-trained, and then the whole network is trained. In the pre-training process, only the generator is trained with L1 loss of the output image and the original image. The batch size set in training was 1 , the epoch was 300 and filter channels was 128 . The peak signal to noise ratio (PSNR) in the pre-training stage of different Mobilenet was shown in Table 1. The batch size of the overall training was 1, the learning rate was optimized by Adam [20], the initial learning rate was set as $10^{-4}$ and the epoch was set as 250 .

Table 1. The peak signal to noise ratio (PSNR) in the pre-training stage of different Mobilenet. M-v2 is Mobilenet-v2, M-v3-1 is Mobilenet-v3-large, M-v3-1-DSC is Mobilenet-v3-large-DSC and M-v3-s is Mobilenet-v3-small.

\begin{tabular}{|c|c|c|c|c|}
\hline & M-v2 & M-v3-1 & M-v3-1-DSC & M-v3-s \\
\hline PSNR & 23.74 & 23.73 & 23.49 & 23.29 \\
\hline Parameters & $3.31 \mathrm{M}$ & $4.11 \mathrm{M}$ & $3.01 \mathrm{M}$ & $2.13 \mathrm{M}$ \\
\hline
\end{tabular}

\section{EXPERIMENTAL ANALYSIS}

This section mainly introduces the quality evaluation method of the deblurred images, reasoning efficiency and computational cost. A new measurement index model was proposed as a trade-off to measure the cost performance of the model, to weigh the loss of precision and speed with the increase of computational cost. Moreover, several existing supervised methods and some new proposed methods were compared experimentally with GoPro Dataset.

\section{A. Evaluation Method}

The traditional image recovery quality evaluation indexes are peak signal to noise ratio (PSNR) and structural similarity (SSIM). In this paper, we also use target detection to evaluate the recovery quality, to evaluate the ability of the recovery model to deal with practical problems. The YOLO network was used to obtain precision and recall rate for target detection evaluation.

The aim of this paper is to reduce reasoning time and calculation cost at a proper expense of accuracy. However, with the reduction of reasoning time and calculation cost, the cost of precision loss needs to be weighed. Therefore, this paper proposed a method to judge whether the modification of the model has value based on whether the compromise rate is greater than 0 . Reasoning time is not proportional to computational cost; some networks such as EfficientNet have a low FLOP but have a higher reasoning time. This is because it uses a lot of high-data read and write operations while reducing the FLOP. As a result, the model spends a lot of time in reading and writing data from video memory, and the GPU's computing power cannot be fully utilized. Therefore, this paper evaluates the compromise rate of reasoning time and calculation cost separately.

Reasoning time compromise rate. Under normal circumstances, the requirement of reasoning efficiency is to achieve real-time processing, which requires the reasoning efficiency to be improved by several times. So in this paper, we built a reasoning time compromise rate formula to evaluate the trade-off, as shown in Eq.2. The value $\alpha$ is set according to the specific tasks of real-time demand, $P$ represents parameters 
before the modification of the network, $\mathrm{A}$ is the parameters of the modified network.

$$
\text { trade-off - time }=\left(A_{P S N R}-P_{P S N R}\right)+\alpha \frac{P_{\text {time }}}{A_{\text {time }}}
$$

Calculation cost compromise rate. Reducing the operation cost usually can reduce the demand of equipment work force, the gap between hardware memory is usually an exponential of 2. Therefore, we construct a calculation cost compromise rate formula for evaluating. As shown in Eq.3, where $\beta$ is calculated according to the specific task to reduce the calculation cost requirement, $\mathrm{P}$ is the parameter before the network modification; $\mathrm{A}$ is the parameter after the network modification.

$$
\begin{array}{r}
\text { trade }- \text { off }- \text { flops }=\left(A_{P S N R}-P_{P S N R}\right)+ \\
\beta\left(\log _{2} p_{\text {time }}-\log _{2} A_{\text {time }}\right)
\end{array}
$$

\section{B. Experimental Comparison}

We compared the latest several supervised blurred image restoration networks on GoPro data set, the PSNR and SSIM index values of different methods are shown in Table 2. The SRN method has the highest PSNR value, mainly because its losses are calculated by entire mean square error. The DeBlurganV2-Inception has the highest SSIM value.

Table 2. Comparison of PSNR and SSIM indexes of different methods.

\begin{tabular}{|l|c|c|c|c|c|c|c|}
\hline \multirow{2}{*}{} & \multirow{2}{*}{ SRN } & \multirow{2}{*}{ DeblurGAN } & \multicolumn{2}{|c|}{ DeblurGANv2 } & \multicolumn{3}{|c|}{ Proposed } \\
\cline { 4 - 8 } & & & Inception & Mobilenet-v2 & v3_large & v3_large_DSC & v3_small \\
\hline PSNR & $\mathbf{2 9 . 9 7}$ & 28.39 & 29.18 & 26.89 & 26.66 & 26.21 & 25.99 \\
\hline SSIM & 0.932 & 0.926 & $\mathbf{0 . 9 3 4}$ & 0.903 & 0.902 & 0.901 & 0.898 \\
\hline
\end{tabular}

The object detection recall rate and accuracy of different methods are shown in Table 3. The clear image is taken as the accuracy and recall rate of the method respectively. Deblurganv2-Inception method showed the highest accuracy ground truth. Rec. and Pre. in the table represent the detection

and recall rate.

Table 3. Object detection results comparison of different methods.

\begin{tabular}{|c|c|c|c|c|c|c|c|c|}
\hline \multicolumn{2}{|c|}{} & \multirow{2}{*}{ Blurred } & \multirow{2}{*}{ DeblurGAN } & \multicolumn{2}{c|}{ DeblurGANv2 } & & \multicolumn{2}{c|}{ Proposed } \\
\cline { 5 - 10 } & & & Inception & Mobilenetv2 & v3_large & v3_large_DSC & v3_small \\
\hline \multirow{3}{*}{ YOLO } & Rec. & 0.369 & 0.791 & $\mathbf{0 . 8 8 2}$ & 0.813 & 0.806 & 0.764 & 0.731 \\
\cline { 2 - 10 } & Pre. & 0.796 & 0.781 & $\mathbf{0 . 9 3 2}$ & 0.774 & 0.782 & 0.783 & 0.782 \\
\hline
\end{tabular}

The comparisons of reasoning efficiency and computational cost are shown in Table 3. In our experiments, we used GeForce GTX 1050Ti GPU with 4G video memory to test the reasoning time, which has less computing power than the NVIDIA GTX Titan X with $12 \mathrm{G}$ video memory used in DeBlurGan-v2 [11]. The reasoning time for DeBlurganV2-Inception network on NVIDIA GTX Titan X

was $0.35 \mathrm{~s}$, and the time for DeBlurganV2-Mobilenet was $0.06 \mathrm{~s}$, which can almost meet the real-time video processing requirements. As shown in Table 4, the method proposed in this paper has advantages in reasoning time and computing cost, especially when using Mobilenetv3_large_DSC model as the backbone network, which has the fastest reasoning efficiency and the lowest computing cost.

Table 4. Comparison of reasoning efficiency and computational cost of different methods.

\begin{tabular}{|c|c|c|c|c|c|c|c|}
\hline \multirow{2}{*}{} & \multirow{2}{*}{ SRN } & \multirow{2}{*}{ DeblurGAN } & \multicolumn{2}{|c|}{ DeblurGANv2 } & \multicolumn{3}{c|}{ Proposed } \\
\cline { 4 - 8 } & & & Inception & Mobilenetv2 & v3_large & v3_large_DSC & v3_small \\
\hline TIME & $3.83 \mathrm{~s}$ & $2.07 \mathrm{~s}$ & $1.52 \mathrm{~s}$ & $1.22 \mathrm{~s}$ & $0.91 \mathrm{~s}$ & $\mathbf{0 . 7 2}$ & $0.80 \mathrm{~s}$ \\
\hline FLOPs & $1434.82 \mathrm{G}$ & $678.29 \mathrm{G}$ & $411.34 \mathrm{G}$ & $43.75 \mathrm{G}$ & $39.27 \mathrm{G}$ & $\mathbf{8 . 8 3 G}$ & $31.10 \mathrm{G}$ \\
\hline
\end{tabular}

Compromise ratio of different methods is shown in Table 5 , the results of DeblurGANv2 is calculated in contrast to
DeblurGAN. Results of the proposed network is calculated in contrast to the compromise ratio of DeblurGANv2_Mobilenetv2.

Table 5. Comparison of compromise rate between different methods

\begin{tabular}{|c|c|c|}
\hline & DeblurGANv2 & Proposed \\
\hline
\end{tabular}




\begin{tabular}{|l|c|c|c|c|c|}
\hline & Inception & Mobilenetv2 & v3_large & v3_large_DSC & v3_small \\
\hline Trade-off-time & $0.79+1.36 \alpha$ & $-1.50+1.69 \alpha$ & $-0.23+1.34 \alpha$ & $-0.68+1.69 \alpha$ & $-0.9+1.53 \alpha$ \\
\hline Trade-off-FLOPs & $0.79+0.73 \beta$ & $-1.50+3.87 \beta$ & $-0.23+0.24 \beta$ & $-0.68+2.4 \beta$ & $-0.9+0.54 \beta$ \\
\hline
\end{tabular}

As shown in Fig. 5, if $\alpha$ is setted greater than 0 , the DeblurGANv2_Inception network's time compromise rate is greater than 0 and is far ahead of other methods due to its higher accuracy and reasoning efficiency. When $\alpha$ is set as a small value, that is, when applied to high-precision tasks, the time compromise rate of other methods is less than 0 . When applied to tasks requiring high efficiency of reasoning, v3_large_DSC approach proposed in this paper has a higher time compromise rate than DeblurGANv2_Inception and DeblurGANv2_Mobilenetv2 when $\alpha$ increases.

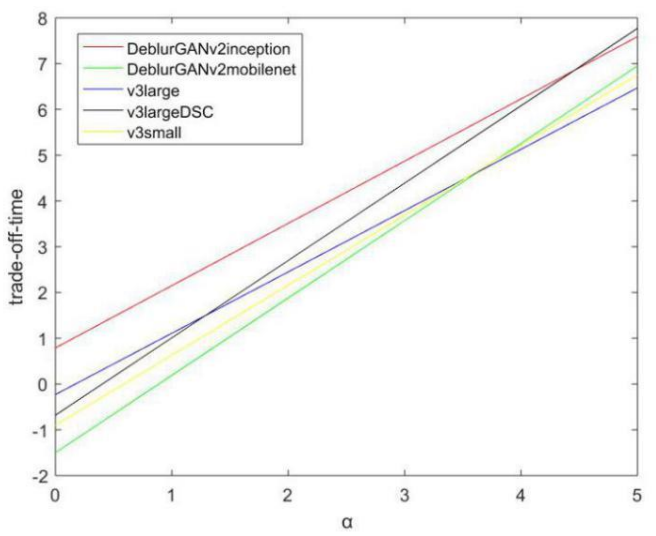

Fig. 5. Compromise ratio of time

The compromise rate of operation cost is shown in Fig.6, as long as the value $\beta$ is greater than 0 , the calculation cost compromise rate of DeblurGANv2 Inception network is greater than 0 due to its higher accuracy and promotion. When applied to high-precision tasks where $\beta$ is small, the compromise rate of operation cost of other methods is less than 0 . When Bincreases, DeblurGANv2_Mobilenetv2 approach is far ahead than other methods when used on computation-intensive hardware, and the v3_large_DSC approach proposed in this paper exceeds DeblurGANv2_Inception.

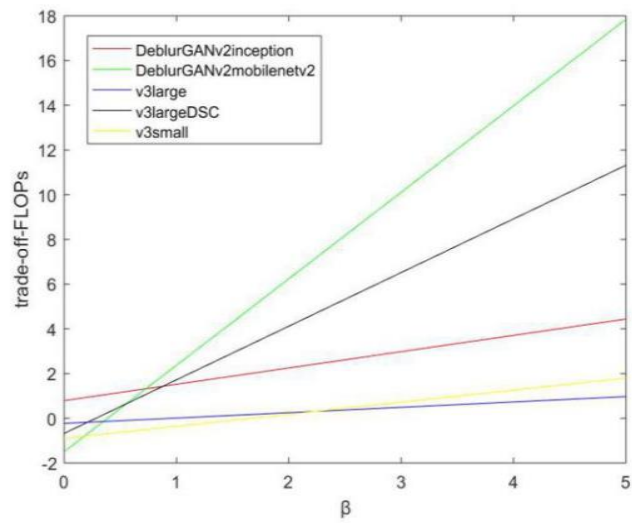

Fig. 6. Calculation cost compromise rate

\section{CONCLUSION}

In the field of image processing, motion blurred image deblurring is one of the most basic research topics. With the widespread application of motion blur recovery, its research trend is not limited to obtain better recovery result, but also to pursue higher reasoning efficiency and lower computational cost. In this paper, the main contributions are as follows: (1) Improved the latest DeBlurgan-v2 method by modifying the feature extraction backbone network for mobilenet-V3 network which has a higher accuracy and reasoning efficiency. (2)A new evaluation method, the compromise rate which can weigh the calculation time with image recovery precision is proposed, facilitating the evaluation of whether the modification to the network is valuable.

In future study, more precise image deblurring methods with higher reasoning efficiency and lower computational cost would be an important research direction. Besides, GAN based deep neural networks for non-uniform blurred image recovery can be another intersting problem to explore.

\section{References}

[1] O. Kupyn, V. Budzan, M. Mykhailych, D. Mishkin and J. Matas, "DeblurGAN: Blind Motion Deblurring Using Conditional Adversarial Networks," 2018 IEEE/CVF Conference on Computer Vision and Pattern Recognition, Salt Lake City, UT, 2018, pp. 8183-8192, doi: 10.1109/CVPR.2018.00854.

[2] R. Fergus, B. Singh, A. Hertzmann, S. T. Roweis, and W. T.Freeman. Removing camera shake from a single photograph.ACM Trans. Graph., 25(3):787-794, July 2006. 2, 5

[3] S. D. Babacan, R. Molina, M. N. Do, and A. K. Katsaggelos.Bayesian blind deconvolution with general sparse image priors.In European Conference on Computer Vision (ECCV),Firenze, Italy, October 2012. Springer.

[4] L. Xu and J. Jia. Two-phase kernel estimation for robust motion deblurring. In Lecture Notes in Computer Science (including subseries Lecture Notes in Artificial Intelligence and Lecture Notes in Bioinformatics), 2010.

[5] L. Xu, J. S. J. Ren, C. Liu, and J. Jia. Deep convolutional neural network for image deconvolution. In Proceedings of the 27th International Conference on Neural Informatin Processing Systems - Volume 1, NIPS'14, pages 1790-1798,Cambridge, MA, USA, 2014. MIT Press.

[6] L. Xu, S. Zheng, and J. Jia. Unnatural L0 Sparse Representation for Natural Image Deblurring. 2013.

[7] I. J. Goodfellow, J. Pouget-Abadie, M. Mirza, B. Xu,D.Warde-Farley, S. Ozair, A. Courville, and Y. Bengio. Generative Adversarial Networks. June 2014.

[8] M. Arjovsky, S. Chintala, and L. Bottou. Wasserstein GAN.ArXiv e-prints, Jan. 2017.

[9] P. Isola, J.-Y. Zhu, T. Zhou, and A. A. Efros. Image-to-image translation with conditional adversarial networks. arxiv, 2016.

[10] I. Gulrajani, F. Ahmed, M. Arjovsky, V. Dumoulin, and A. Courville. Improved Training of Wasserstein GANs. ArXiv e-prints, Mar. 2017.

[11] Kupyn, Orest et al. DeblurGAN-v2: Deblurring (Orders-of-Magnitude) Faster and Better. 2019 IEEE/CVF International Conference on Computer Vision (ICCV) (2019): 8877-8886.

[12] Tsung-Yi Lin, Piotr Doll'ar, Ross Girshick, Kaiming He, Bharath Hariharan, and Serge Belongie. Feature pyramid networks for object detection. In Proceedings of the IEEE Conference on Computer Vision and Pattern Recognition,pages 2117-2125, 2017.

[13] Howard, Andrew G, et al. "MobileNets: Efficient Convolutional Neural Networks for Mobile Vision Applications." (2017). 
[14] Christian Szegedy, Sergey Ioffe, Vincent Vanhoucke, and Alexander A Alemi. In Thirty-First AAAI Conference on Artificial Intelligence, 2017.

[15] Mark Sandler, Andrew Howard, Menglong Zhu, Andrey Zhmoginov, and Liang-Chieh Chen. MobileNetV2: Inverted Residuals and Linear Bottlenecks. arXiv e-prints, page, arXiv:1801.04381, Jan 2018.

[16] Howard, A. , Sandler, M. , Chen, B. , Wang, W. , Chen, L. C. , \& Tan, M. , et al. (2020). Searching for MobileNetV3. 2019 IEEE/CVF International Conference on Computer Vision (ICCV). IEEE.

[17] Xudong Mao, Qing Li, Haoran Xie, Raymond Y. K. Lau, and Zhen Wang. Least squares generative adversarial networks,2016. cite arxiv: 1611.04076 .

[18] S. Nah, T. Hyun, K. Kyoung, and M. Lee. Deep Multi-scale Convolutional Neural Network for Dynamic Scene Deblurring.2016.

[19] S.Niklaus, L. Mai, and F. Liu. Video frame interpolation via adaptive separable convolution[C].In Proceedings of the IEEE International Conference on Computer Vision, pages 261-270, 2017.

[20] D. P. Kingma and J. Ba. Adam: A method for stochastic optimization. CoRR, abs/1412.6980, 2014.

[21] M. Tan and Q. V. Le. EfficientNet: Rethinking Model Scaling for Convolutional Neural Networks[C].ICM

[22] A. Chakrabarti. A neural approach to blind motion deblurring[C].In Lecture Notes in Computer Science (including sub-series Lecture Notes in Artificial Intelligence and Lecture Notes in Bioinformatics), 2016.

[23] O. Kupyn, V. Budzan, M. Mykhailych, D. Mishkin, and J. Matas. Deblurgan: Blind motion deblurring using conditional adversarial networks[C].In Proceedings of the IEEE Conference on Computer Vision and Pattern Recognition, 2018.

[24] D. Gong, J. Yang, L. Liu, Y. Zhang, I. Reid, C. Shen, A. V. D. Hengel, and Qinfeng Shi. From Motion Blur to Motion Flow: a Deep Learning Solution for Removing Heterogeneous Motion Blur[C].In Proceedings of the IEEE Conference on Computer Vision and Pattern Recognition,2016.

[25] L. Li, J. Pan, W. Lai, C. Gao, N. Sang, M. Yang. Learning a Discriminativ-e Prior for Blind Imag-e Deblurring[C].IEEE International Conference on Computer Vision,2018.

[26] D. Zoran, and Y. Weiss. From learning models of natural image patches to whole image restoration[C].In ICCV, pages 479-486. IEEE, 2011.

[27] W.-S. Lai, J.-J. Ding, Y .-Y . Lin, and Y .-Y . Chuang. Blur kernel estimation using normalized color-line prior[C].In IEEE Conference on Computer Vision and Pattern Recognition,2015.

[28] C. J. Schuler, M. Hirsch, S. Harmeling, and B. Schölkopf. Learning to de-blur[C].IEEE transactions on pattern analysis and machine inte lligence, 38(7):1439-1451, 2016.

[29] S. Ramakrishnan, S. Pachori, A. Gangopadhyay, and S. Ra-man. Deep generative filter for motion debl-urring[C].IEEE International Conference on Computer Vision Work-shops (ICCVW), pages 2993-3000, 2017.

[30] P. Isola, J.-Y. Zhu, T. Zhou, and A. A. Efros. Image to-image translation with conditional adversarial networks[J].arxiv, 2016.

[31] O.Kupyn, T.Martyniuk, J.Wu, Z.Wang. DeblurGAN-v2: Deblurring (Orders-of-Magnitude) Faster and Better[C].In Proceedings of the IEEE Conference on Computer Vision and Pattern Recognition, 2019.

[32] X. Tao, H. Gao, X. Shen, J. Wang, and J. Jia. Scale-recurrent network for deep image deblurring[C].In Proceedings of the IEEE Conference on Computer Vision and Pattern Recognition, pages 8174-8182, 2018.

[33] B. Lu, J. -C. Chen, R. Chellappa. Unsupervised Domain-Specific Deblurring via Disentangled Representations [J]. arXiv:1903.01594v1, 5 Mar 2019.

[34] H.-Y. Lee, H.-Y. Tseng, J.-B. Huang, M. Singh, and M.-H.Yang. Diverse image-to-image translation via disentangled representations[C].In Proceedings of European Conference on Computer Vision (ECCV), pages 36-52. Springer, 2018.

[35] C. Szegedy, S. Ioffe, V. Vanhoucke, and A. A Alemi. Inception-v4, inception-resnet and the impact of residual connections on learning[C].In Thirty-First AAAI Conference on Artificial Intelligence, 2017.

[36] T. Madam Nimisha, K. Sunil, and A. Rajagopalan. Unsupervised class-specific deblurring[C].In Proceedings of the European Conference on Computer Vision, pages 353369, 2018.

[37] M. Sandler, A. Howard, M. Zhu, A. Zhmoginov, and L. -C. Chen MobileNetV2: Inverted Residuals and Linear Bottlenecks[J].arXiv e-prints, page arXiv:1801.04381, Jan 2018

[38] T.-Y. Lin, P. Doll'ar, R. Girshick, K. He, B. Hariharan, and S. Belongie. Feature pyramid networks for object detection[C].In Proceedings of the IEEE Conference on Computer Vision and Pattern Recognition, pages $2117-2125,2017$

[39] Mario Milicevic, Krunoslav Zubrinic, Ines Obradovic, Tomo Sjekavica, Data Augmentation and Transfer Learning for Limited Dataset Ship
Classification, WSEAS Transactions on Systems and Control, pp. 460-465, Volume 13, 2018.

[40]Ki-Hyun Jung, Comparative Histogram Analysis of LSB-based Image Steganography, WSEAS Transactions on Systems and Control, pp. 103-112, Volume 13, 2018.

Ning Li, Ph.D in Engineering, director of experimental center, senior experimenter, main research areas include mechanical design and AI.

Songnan Chen, Ph.D. Candidate in forestry engineering, main research areas include computer vision and Artificial intelligence.

Mengxia Tang, Master Student in forestry engineering, main research areas includes computer vision and artificial intelligence.

Jiangming Kan, professor, Ph.D in Engineering, main research areas include forestry equipment and informatization and computer Vision.

\section{Creative Commons Attribution License 4.0 (Attribution 4.0 International , CC BY 4.0)}

This article is published under the terms of the Creative Commons Attribution License 4.0 https://creativecommons.org/licenses/by/4.0/deed.en US 